\title{
Resolving the Disk-Halo Degeneracy using Planetary Nebulae
}

\author{
S. Aniyan ${ }^{1}$, K. C. Freeman ${ }^{1}$, M. Arnaboldi ${ }^{2}$, O. Gerhard ${ }^{3}$, \\ L. Coccato ${ }^{2}$, M. Fabricius ${ }^{3}$, K. Kuijken ${ }^{4}$ and M. Merrifield ${ }^{5}$ \\ ${ }^{1}$ Research School of Astronomy \& Astrophysics, Australian National University \\ email: suryashree.aniyan@anu.edu.au \\ ${ }^{2}$ European Southern Observatory, Garching \\ ${ }^{3}$ Max-Planck-Institut fur Extraterrestrische Physik, Garching \\ ${ }^{4}$ Leiden Observatory, Leiden University \\ ${ }^{5}$ School of Physics and Astronomy, University of Nottingham
}

\begin{abstract}
The decomposition of the $21 \mathrm{~cm}$ rotation curve of galaxies into contribution from the disk and dark halo depends on the adopted mass to light ratio $(M / L)$ of the disk. Given the vertical velocity dispersion $\left(\sigma_{z}\right)$ of stars in the disk and its scale height $\left(h_{z}\right)$, the disk surface density and hence the $M / L$ can be estimated. Earlier works have used this technique to conclude that galaxy disks are submaximal. Here we address an important conceptual problem: star-forming spirals have an old (kinematically hot) disk population and a young cold disk population. Both of these populations contribute to the integrated light spectra from which $\sigma_{z}$ is measured. The measured scale height $h_{z}$ is for the old disk population. In the Jeans equation, $\sigma_{z}$ and $h_{z}$ must pertain to the same population. We have developed techniques to extract the velocity dispersion of the old disk from integrated light spectra and from samples of planetary nebulae. We present the analysis of the disk kinematics of the galaxy NGC 628 using IFU data in the inner regions and planetary nebulae as tracers in the outer regions of the disk. We demonstrate that using the scale height of the old thin disk with the vertical velocity dispersion of the same population, traced by PNe, results in a maximal disk for NGC 628. Our analysis concludes that previous studies underestimate the disk surface mass density by $\sim 2$, sufficient to make a maximal disk for NGC 628 appear like a submaximal disk.
\end{abstract}

Keywords. galaxies: kinematics and dynamics, galaxies: spiral, galaxies: halos

\section{Introduction}

Decomposing the rotation curve of spiral galaxies into contribution from the disk and the dark halo provides a direct technique for studying the structure of the dark halos. The decomposition depends however on the adopted $M / L$ of the stellar disk (van Albada et al. 1985) which is still uncertain. Adopting different $M / L$ values can result in a maximal $\dagger$ or a submaximal disk, both of which can fit the observed rotation curves equally well (but with very different dark halos). The $M / L$ value is therefore critical to obtaining the parameters of the dark halos, such as their scale densities and scale lengths. These quantities are cosmologically significant, because the densities and scale radii of dark halos follow well-defined scaling laws and can be used to measure the redshift of assembly of halos of different masses (Kormendy and Freeman 2016).

Several techniques have been used to break the disk-halo degeneracy but they all present challenges. One of the more direct methods to break this degeneracy uses the

$\dagger$ A maximal disk has the maximum $M / L$ value consistent with the observed rotation curve and a non-hollow dark halo. Typically, the disk of a maximal disk provides about $85 \%$ of the rotational velocity at the peak of the rotation curve (Sackett 1997). 
vertical velocity dispersion of tracers in the disks to measure the surface mass density of the disk (e.g. Bottema 1997). Using the 1D Jeans equation in the vertical direction, the vertical luminosity-weighted velocity dispersion $\sigma_{z}$ and the vertical exponential disk scale height $h_{z}$ together give the surface mass density $\Sigma$ of the disk via the relation:

$$
\Sigma=f \sigma_{z}^{2} / G h_{z}
$$

where $\mathrm{G}$ is the gravitational constant and $f$ is a geometric factor, known as the vertical structure constant, that depends weakly on the adopted vertical structure of the disk. The surface brightness of the disk and the surface mass density together give the $M / L$ of the disk, which is needed to break the disk-halo degeneracy.

The scale height $h_{z}$ of the thin disk cannot be measured directly for face-on galaxies. Studies of edge-on disk galaxies show a correlation between the scale height and indicators of the galaxies' mass scale, such as the absolute magnitude and the circular velocity (eg Yoachim \& Dalcanton 2006). We can, therefore, estimate the scale height statistically using other known features of the galaxy.

The other parameter, $\sigma_{z}$ of the disk, can be measured either from: (1) spectra of the integrated light of the disk in relatively face-on galaxies, or (2) the velocity distribution of a population of stellar tracers (such as planetary nebulae).

The early dynamical estimates of vertical velocity dispersion in external galaxies (e.g. Bottema 1997) indicated that the disks are submaximal, consistent with more recent estimates. The DiskMass Survey (Bershady et al. 2011) used integral-field spectroscopy to measure the stellar kinematics of the disks of near face-on galaxies and find that the dynamical stellar $M / L$ obtained from the surface mass density is about 3 times lower than the $M / L$ from maximum disk hypothesis and conclude that disks are submaximal. Work by Herrmann \& Ciardullo (2008, 2009a, 2009b) looked at 5 near-face-on spirals (including our target galaxy) using PNe as tracers and found that in the later-type (Scd) systems, the disks are clearly sub-maximal. In earlier ( $\mathrm{Sc}$ ) galaxies, this discrepancy is smaller but still present; only the very-early Sab system M94 has evidence for a maximal disk.

An important conceptual problem has, however, been overlooked in the earlier studies described above. Eqn. 1.1 comes from the vertical Jeans equation for an equilibrium disk. It is therefore essential that the vertical disk scale height $h_{z}$ and the vertical velocity dispersion $\sigma_{z}$ should refer to the same population of stars.

The red and near-infrared measurements of $h_{z}$ of edge-on disk galaxies are dominated by the red giants of the older, kinematically hotter population, away from the dust plane. On the other hand, $\sigma_{z}$ is usually measured from integrated light spectra near the $\mathrm{Mg}$ b lines $(\sim 5150-5200 \AA)$, since this region has many absorption lines and the sky is relatively dark. The disks of star-forming spirals include a population of young (ages $<2$ Gyr), kinematically cold stars among a population of older, kinematically hotter stars. The red giants of this mixed young + old population provide most of the absorption line signal that is used for deriving velocity dispersions from the integrated light spectra of galactic disks.

Therefore, in Eqn. 1.1, we should be using the $\sigma_{z}$ of the older disk stars in combination with the $h_{z}$ of this same population for an accurate determination of the surface mass density. However, because of limited signal-to-noise ratio (SNR) of previous studies, a single kinematical population is adopted for the velocity dispersion whereas, ideally, the dispersion of the older stars should be extracted from the composite observed spectrum of the younger and older stars.

Adopting a single kinematical population for a composite kinematical population gives a $\sigma_{z}$ that is smaller than the dispersion of the old disk giants (for which the $h_{z}$ was 
measured), and hence underestimates the surface density of the disk. A maximal disk will then appear submaximal. A recent study of the K-giants in the solar neighbourhood by Aniyan et al. (2016) showed that the young stars contribute significantly to the total light and that the velocity dispersion derived assuming a single population of tracers leads to the disk surface mass density being underestimated by a factor $\sim 2$.

We present the analysis of the disk kinematics of the galaxy NGC 628. We demonstrate that using the scale height of the old thin disk with the vertical velocity dispersion of the same population, will result in a maximal disk for NGC 628.

\section{Observations and Data Analysis}

We look at the face-on galaxy (inclination $\sim 6.5^{\circ}$ ) NGC 628. Two complementary analyses were carried out: (1) integral light spectroscopy using the VIRUS-W instrument at McDonald observatory, and (2) using the Planetary nebula spectrograph (PN.S) instrument at La Palma to identify PNe in the outer regions of the disk.

The VIRUS-W is an optical-fibre-based Integral Field Unit (IFU) spectrograph at the 2.7m Harlan J. Smith Telescope at McDonald Observatory in Texas. The IFU has 267 fibres with a spectral resolution of $\mathrm{R} \sim 8700$. The spectral coverage is from $4802-5470$ $\AA$. The VIRUS-W observations were carried out in October 2014. The IFU was placed at several positions around a radius of $\sim 1$ scale length. The spectrum from each fibre was summed up to build the SNR of the final spectrum. We thus end up with a spectrum that is representative of the kinematics of the galaxy at a mean luminosity weighted radius of $78^{\prime \prime}$. We used the pPXF code (Cappellari \& Emsellem 2004) to retrieve the line of sight dispersions $\left(\sigma_{L O S}\right)$ of the kinematically hotter and colder stellar components of the thin disk. We then calculated the $\sigma_{z}$ from the $\sigma_{L O S}$ for both components, with conventional assumptions about the anisotropy of the velocity dispersion.

The planetary nebula spectrograph (PN.S) is an imaging spectrograph designed for efficient observation of extragalactic PNe, that operates on the $4.2 \mathrm{~m}$ William Herschel Telescope at La Palma. The PN.S has a 'left' and 'right' arm in which the light is dispersed in opposite directions. Combining these two counter-dispersed images allows the PNe to be detected and their radial velocities to be measured in a single observation. The data for NGC 628 were acquired in September 2014. We obtained 14 images centred on the centre of the galaxy, each with an exposure time of 1800s. These 14 left and right images were stacked to create the final galaxy image with high SNR. For this study, sextractor was used to identify [OIII] emitters in the stacked left and right image. As an added check, we also performed a visual inspection of the identified sources, to reject any obvious extended sources and add any faint point-like objects that the code may have missed. This resulted in a catalogue of 716 point sources identified in the left and right arm of NGC 628. Figure 1 shows a region of identified sources on the image from the left arm. However, this sample is a mixture of HII regions and PNe, since both can have strong [OIII] emissions. We calculated the bright luminosity cut-off for this galaxy and only include the objects fainter than this cut-off in our analysis. This excludes most of the obvious, bright resolved HII regions. The objects were then put into radial bins with 155 objects in each bin. The dispersions for the hot and cold components in each bin were then calculated using a maximum likelihood estimator code written in python.

\section{Results and Discussion}

We used integrated light data from the VIRUS-W spectrograph and PNe velocities from the PN.S instrument to extract the velocity dispersion of the disk. We extract two $\sigma_{z}$ values: one representing the kinematically cold disk and another representing the kinematically hot disk of NGC 628. We compared our values of the hot and cold component 


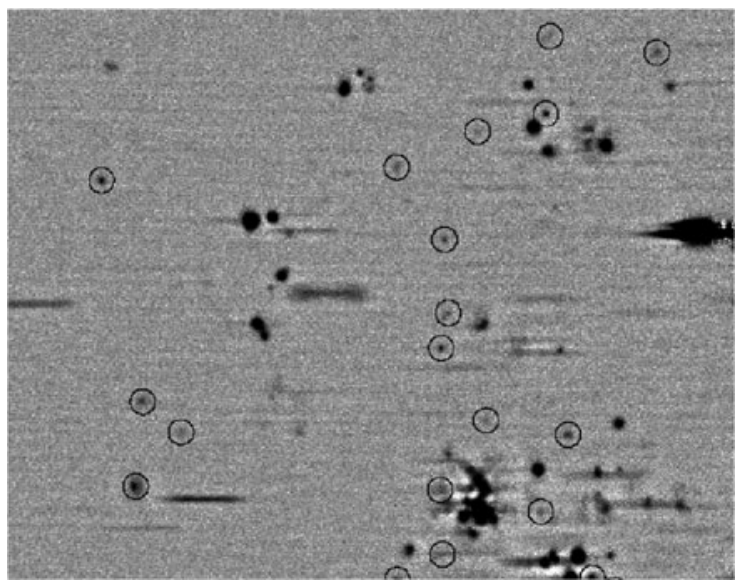

Figure 1. A part of the field in the image from the left arm of the planetary nebula spectrograph showing the identified planetary nebulae. The horizontal streaks are the spectra of stars in the field, limited in wavelength by the bandpass of the narrow band [OIII] filter.

vertical velocity dispersion with the results from Herrmann \& Ciardullo (2009b), who assumed a single population of $\mathrm{PNe}$ and did not separate out the contribution of the cold and hot components. The one-component fit to our data agrees fairly well with the results from Herrmann \& Ciardullo (2009b). Our two-component fit shows a hotter old disk population, with a velocity dispersion that follows the expected radial exponential decline with twice the galaxy's radial scale length, indicating that NGC 628 has an $M / L$ ratio that is approximately constant with radius.

We use the calculated $\sigma_{z}$ values to determine the disk surface mass density at each radial bin. Our central disk surface mass density is a factor of $\sim 2$ times larger than the value obtained by Herrmann \& Ciardullo (2009b). We use BVRI photometry from the literature to calculate the $M / L$ in different bands in this galaxy. We do, in fact, get a constant $M / L$ value for NGC 628 , and our $M / L$ values are about twice those found by Herrmann \& Ciardullo (2009b). This galaxy appears to have a near-maximal disk.

This is the first report from a larger study of nearby face-on spirals, using a combination of integrated light and PNe tracers. The analysis of these galaxies is currently underway.

\section{Acknowledgements}

The authors would like to thank the staff at McDonald Observatory and WHT for providing technical support during observations. SA would like to thank the SOC for granting an oral contribution to present this work, and for awarding a prize for this talk.

\section{References}

Aniyan, S. et al. 2016, MNRAS, 456, 1484

Bershady, M. A. et al. 2011, ApJ, 739, L47

Bottema, R. 1997, A \& A 328, 517

Cappellari, M. \& Emsellem, E. 2004, PASP, 116, 138

Herrmann, K. A., Ciardullo, R., Feldmeier, J. J., \& Vinciguerra, M. 2008, ApJ, 683, 630

Herrmann, K. A. \& Ciardullo, R. 2009, ApJ, 703, 894

Herrmann, K. A. \& Ciardullo, R. 2009, ApJ, 705, 1686

Kormendy, J. \& Freeman, K. C. 2016, ApJ 817, 84

Sackett, P. D. 1997, ApJ 483, 103

van Albada, T. S., Bahcall, J. N., Begeman, K., \& Sancisi, R. 1985, ApJ, 295, 305

Yoachim, P. \& Dalcanton, J. J., 2006, AJ, 131, 226 